\title{
Planejamento de trajetórias polinomiais para robótica com Arduino
}

\section{Polynomial trajectory planning for robotics with Arduino}

\author{
Naylene Fraccanabbia \\ Instituto Federal de Educação, Ciência e Tecnologia do Rio Grande do Sul (IFRS) \\ Campus Bento Gonçalves, RS, Brasil \\ nayfraccanabbia@outlook.com
}

André Luvisa

Instituto Federal de Educação, Ciência e Tecnologia do Rio Grande do Sul (IFRS)

Campus Bento Gonçalves, RS, Brasil

andreluvisa@yahoo.com.br

Delair Bavaresco

Instituto Federal de Educação, Ciência e Tecnologia do Rio Grande do Sul (IFRS)

Campus Bento Gonçalves, RS, Brasil delair.bavaresco@bento.ifrs.edu.br

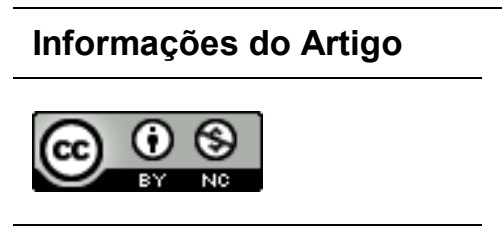

Histórico do Artigo

Submissão: 31 de março de 2018.

Aceite: 14 de junho de 2018.

Palavras-chave

Tracker

Scilab

Servo Motor

\section{Resumo}

O presente trabalho apresenta resultados de um processo investigatório aplicado ao planejamento de trajetórias polinomiais para robótica com Arduino. Imersa na atmosfera da Quarta Revolução Industrial, a pesquisa ancora-se nos pressupostos teórico metodológicos de "Aprendizagem Baseada em Projetos", em uma estreita interligação com a proposta STEM, como um acrônimo das palavras Science (Ciências), Tecnology (tecnologia), Engineering (Engenharia) e Math (Matemática). O desenvolvimento da pesquisa contou com a utilização de diversos recursos tecnológicos para construção de peças, para filmagem dos experimentos e para captura e processamento de dados e geração de gráficos. Juntamente com a implementação de uma bancada experimental de testes, a pesquisa permitiu avaliar o desempenho de diferentes estratégias de geração de trajetórias para o deslocamento de uma haste fixada ao eixo de rotação de um Micro Servo Motor utilizado para acionamento em robótica com Arduino. Os resultados obtidos mostram que a estratégia de suavização de trajetórias de deslocamento contínuo com a utilização de funções polinomiais é um potencial recurso para a minimização de erros, principalmente para o seguimento de trajetória contínua. A pesquisa realizada por meio do desenvolvimento de projetos experimentais, incentivando a aprendizagem interdisciplinar com foco na aplicação prática do aprendizado, alinha-se com os pressupostos teóricos destacados ao visar o envolvimento dos estudantes em soluções de problemas reais.

\section{Abstract}

The present work presents results of an investigative process applied to the planning of polynomial trajectories for robotics with Arduino. Immersed in the atmosphere of the Fourth Industrial Revolution, the research anchors itself in the theoretical methodological assumptions of ProjectBased Learning, in close connection with the STEM proposal, as an acronym for the words Science, Technology, Engineering and Math. The development of the research counted with the use of several technological resources for construction of pieces, for filming of the experiments and for capture and processing of data and generation of graphs. Along with the implementation of an experimental test bench, the research allowed to evaluate the performance of different strategies of trajectory generation for the displacement of a rod fixed to the axis of rotation of a Micro Servo Motor used for robotic drive with Arduino. The results obtained show that the strategy of smoothing continuous displacement trajectories with the 
use of polynomial functions is a potential resource for the minimization of errors, mainly for continuous trajectory tracking. The research carried out through the development of experimental projects, encouraging interdisciplinary learning focusing on the practical application of learning, is aligned with the theoretical assumptions highlighted when aiming the students' involvement in solutions of real problems.

\section{Introdução}

Estamos vivendo a quarta Revolução Industrial. Uma era de crescentes mudanças tecnológicas, as quais implicam mudanças sociais e passam a nortear nossa forma de vida. É o que afirma Klaus Schwab (2016), autor do livro intitulado "A Quarta Revolução Industrial". Para o autor,

estamos no início de uma revolução que alterará profundamente a maneira como vivemos, trabalhamos e nos relacionamos. Em sua escala, escopo e complexidade, a quarta revolução industrial é algo que considero diferente de tudo aquilo que já foi experimentado pela humanidade (SCHWAB, 2016, p. 11).

Esse momento acontece após três processos históricos transformadores. O primeiro foi a mecanização da produção entre 1760 e 1840. Posteriormente, a partir de 1850, a eletricidade permitiu a manufatura em massa. O terceiro processo marca a chegada da eletrônica, da tecnologia da informação e das telecomunicações, já no século XX.

Nanotecnologias, neurotecnologias, robôs, inteligência artificial, biotecnologia, sistemas de armazenamento de energia, drones e impressoras 3D são uma síntese das principais ideias acadêmicas relacionadas à Quarta Revolução Industrial, também chamada de Revolução 4.0.

A quarta mudança traz consigo uma tendência à automatização total das fábricas por meio de sistemas ciberfísicos, que foram possíveis graças à internet das coisas e à computação na nuvem. Sanchez (2018) afirma que, inevitavelmente, a Revolução 4.0 gera significativas implicações no sistema educacional escolarizado.

Imaginem vocês que $65 \%$ das crianças que entrarem hoje na escola primária irão trabalhar em empregos que hoje não existem! É urgente uma ação adaptativa de todos e, principalmente, das escolas! [...] Fazendo um paralelo com a nossa escola, concluímos que estamos ainda na versão 2.0. Temos um modelo educacional analógico para uma geração digital, por isto não conseguimos colher bons resultados, pois o serviço prestado não é absorvido pelo aluno (SANCHEZ, 2018, s. p.).

Essa conjuntura impõe a necessidade de definição de estratégias inovadoras para os processos que regem a educação escolarizada. Estratégias que se alinhem com a conjuntura contemporânea de desenvolvimento científico e tecnológico, característico da Revolução 4.0.

Uma abordagem que vem ganhando força em pesquisas contemporâneas é a abordagem STEM. STEM, como acrônimo das palavras Science (Ciências), Tecnology (tecnologia), Engineering (Engenharia) e Math (Matemática) (BYBEE, 2013). Outra, semelhante, é descrita por Project Based Learning (PBL), significando "aprendizagem baseada em projetos", que aposta na construção de conhecimento por meio de um trabalho de investigação que responda a uma pergunta complexa, problema ou desafio (BENDER, 2014). 
Dentre as ideias acadêmicas relacionadas à Revolução 4.0 está a robótica. Esta, por sua vez, apresenta-se como uma possibilidade de interação entre o meio acadêmico e a busca por avanços e por soluções de problemas ligados ao desenvolvimento tecnológico, sobretudo pela potencialidade de criação de mecanismos para a realização de tarefas autônomas. No entanto, historicamente essa abordagem vinha mostrando-se de difícil inserção no meio acadêmico devido ao elevado custo de controladores, atuadores e sensores, necessários para pesquisas acadêmicas em robótica (SILVA, 2011).

Uma potencial alternativa vem ganhando espaço nos últimos anos devido a sua versatilidade de uso e baixo custo de seus componentes. É a plataforma de prototipagem eletrônica Arduino, a qual é uma arquitetura de hardware e software abertos, o que ocasiona baixo custo e facilidade de acesso (ARDUINO, 2018). O Arduino possui uma interface simples, de fácil programação, e com bastante suporte, tanto para os que estão iniciando como também para aqueles que já têm certa experiência. Esse ambiente de desenvolvimento utiliza uma linguagem baseada em linha de código, que tem sintaxe semelhante à linguagem $\mathrm{C}$ e possui ampla documentação a respeito de suas funcionalidades.

Em paralelo, o avanço tecnológico das últimas décadas teve reflexo direto na organização das indústrias, as quais buscam minimizar seus custos industriais através da adoção de diversos modelos de produção. Nesse contexto, a automação vem crescendo por meio da utilização de mecanismos de realização de tarefas autônomas e adaptando-se às necessidades operacionais de determinadas tarefas, empregando garras e/ou ferramentas oportunamente selecionadas (VENTURELLI, 2017). Não obstante, a utilização desses mecanismos requer, na maioria dos casos, controle preciso e movimento suave, evitando erros e comprometimento da execução das tarefas programadas. Para essa finalidade, principalmente na indústria, o planejamento de trajetórias de deslocamento de mecanismos móveis é uma ferramenta importante para o aumento da produtividade através da redução do tempo no processo e da suavização dos movimentos. Com isso é possível evitar colisões e melhorar a precisão de posicionamento e de seguimento de trajetórias dos robôs durante a execução de tarefas.

É nessa atmosfera que se desenvolveu o presente trabalho, o qual tem como objetivo inserir estudantes de graduação em pesquisas aplicadas à robótica educacional e contribuir para o aprimoramento de mecanismos de execução de tarefas autônomas. O problema abordado consiste no planejamento de trajetórias de deslocamento suave para Micro Servo Motores acionados e controlados via plataforma Arduino e na realização de testes de parada e de seguimento de trajetórias.

O Servo Motor é um componente essencial para projetos de robótica, mecatrônica e diversos outros projetos. Na robótica, é responsável por movimentar braços, pernas e mãos dos robôs. No automodelismo, é utilizado para virar as rodas dianteiras dos carrinhos e, no aeromodelismo, é utilizado para controlar os flaps das asas dos aviões. É um atuador muito utilizado em aplicações para 
robótica e, na indústria, é usado em sistemas de automação e, na eletrônica, pode ser usado para movimentos de partes de equipamentos, tais como um porta CDs de um computador.

As tarefas normalmente atribuídas a manipuladores robóticos são classificadas em ponto-aponto e contínuas (POPOV; YUREVICH, 1987). O movimento ponto-a-ponto é utilizado, por exemplo, para a colocação de peças em linhas de produção, e o contínuo, em atividades de lixamento e pinturas.

O problema de geração de trajetória consiste em gerar o histórico temporal da posição para que o mecanismo realize um movimento desejado. O movimento desejado no espaço cartesiano é determinado através dos pontos iniciais e da forma que o caminho deve ser percorrido entre esses pontos e o tempo de percurso. Com isso, a trajetória envolve discussões sobre deslocamento, velocidade e aceleração.

No caso deste estudo, o problema consiste em determinar trajetórias de deslocamento suave, de tal modo que o mecanismo móvel percorra os trechos de deslocamento necessários no tempo planejado, procurando minimizar erros de posicionamento e de seguimento de trajetórias. A estratégia utilizada consiste na determinação de trajetórias polinomiais de diferentes graus, para deslocamento entre dois pontos, nas quais são levadas em consideração a minimização de erros, e a capacidade de processamento exigida para cada caso. A escolha por trajetórias polinomiais se dá porque suas propriedades são bem conhecidas e suas derivadas podem ser facilmente obtidas, resultando também em polinômios.

Os objetivos do trabalho consistem de três etapas: a definição de trajetórias de deslocamento com suavização do movimento nas extremidades, a implementação de testes de parada e de seguimento de trajetórias para mecanismos acionados e controlados por Arduino, e a avaliação dos resultados dos testes. Além delas, são definidas e implementadas estratégias de minimização de erros por meio de manipulação dos polinômios, tais como translação horizontal e vertical, inversão de sentido e deformações que venham a minimizar erros e otimizar o tempo de operação.

$\mathrm{Na}$ busca pelo alcance desses objetivos, os estudantes envolvem-se em um processo de pesquisa, elaboração de hipóteses, busca por recursos e aplicação prática da informação até chegar a uma solução ou produto final. Desse modo, este estudo insere-se em uma perspectiva contemporânea em que a ciência e a tecnologia assumem lugar de destaque na conjuntura socioeconômica globalizada (SANCHEZ, 2018). Nessa perspectiva, a Matemática configura-se como um dos alicerces desse processo, por meio de estratégias de resolução de problemas, modelagem matemática e simulação numérica e, ainda, técnicas de prototipagens e seus processos. 


\section{Referencial Teórico}

O presente trabalho apresenta resultados de uma pesquisa aplicada que se desenvolveu com base em algumas propostas teórico-metodológicas que se adequam ao desenvolvimento de pesquisas em ambientes educacionais. Procurando alinhar-se, também, com as tendências contemporâneas de desenvolvimento científico e tecnológico associado aos sistemas de ensino formal, uma proposta que vem ganhando espaço e adeptos é a proposta STEM, a qual integra os termos Ciência, Tecnologia, Engenharia e Matemática (em inglês). Essa proposta tem a função de interligar as quatro áreas, incentivando a aprendizagem interdisciplinar, com foco na aplicação prática do aprendizado.

O conceito de STEM surgiu em 1890, na Universidade de Chicago, com John Dewey, o qual defendeu a ideia de que a escola deveria adotar o perfil de uma pequena comunidade, ou seja, visava o envolvimento dos estudantes em soluções de problemas reais, as quais se dariam de forma multidisciplinar. A proposta STEM, por interligar as quatro áreas, incentiva os alunos a colaborarem com os docentes e colegas durante o decorrer das aulas, pois possibilita uma maior atenção nas atividades educacionais, visto que pode-se ter aulas diferenciadas, contextualizadas e aplicadas. Como por exemplo, aulas de robótica, projetos interdisciplinares, entre outras. Além disso, nota-se que há uma transferência de aprendizagem de uma situação para outra, nas quais os discentes são desafiados a colocar a mão na massa, criando, planejando, desenvolvendo e implementando projetos que possuam fins essenciais para a vida em sociedade e para seu futuro (ENGLISH, 2016).

Não distante dessa tendência, a proposta de Aprendizagem Baseada em Projetos, em uma tradução para Project Based Learning, defende a ideia de que a formulação do projeto e a realização da pesquisa envolvem a elaboração de um produto final como resultado do trabalho do grupo.

Os estudantes precisam planejar cooperativamente as ações de sua equipe à medida que avançam na solução do problema, desenvolvendo um plano de ação e começando a elaborar uma descrição ou diretrizes para o desenvolvimento de seus produtos ou artefatos [...] Em resumo, um artefato pode ser praticamente qualquer coisa de que o projeto necessite, dada a expectativa de que os artefatos representem coisas necessárias ou usadas no mundo real (BENDER, 2014, p. 17-18).

Em um entendimento similar, Cobb (2003) defende a proposta de Pesquisa de Desenvolvimento em Projetos Experimentais como uma tradução de Design Experiments in Educational Research, introduzida em 1992 por Brown e Collins. Essa perspectiva enfatiza o aprofundamento da compreensão do fenômeno sob investigação pelo pesquisador enquanto a atividade está em andamento, de modo a aplicar diferentes conhecimentos. Na pesquisa de desenvolvimento, os projetos possuem uma abordagem pragmática dupla "- formas particulares de aprendizagem - e uma orientação teórica - desenvolvendo teorias específicas de domínio ao estudar sistematicamente essas formas de aprendizagem e os meios de apoiá-las" (COBB, 2003, p. 9).

Para Doerr e Wood (2006), essa metodologia segue dois princípios norteadores: o desenvolvimento de um processo ou de um produto, e os vários ciclos de análise necessários, com 
a finalidade de aprimorar o produto. No caso deste trabalho, o produto final, mesmo tendo um propósito bem definido, coloca-se em segundo plano. Em se tratando de um ambiente educacional, o processo torna-se a principal ênfase, pois é nele que o aprendizado se torna mais significativo por meio da aplicação dos conceitos teóricos previamente estudados. Segundo Vaz (2004, p. 36), no design experiments "o enfoque está nos significados construídos pelos aprendizes, tendo como objetivo principal analisar o seu pensamento matemático, ou seja, os processos pelos quais esses pensamentos se constroem e suas modificações".

Laboy-Rush (2013) defende a aprendizado baseado em projetos em uma proposta de integração com a metodologia STEM. Para o autor, esse projeto exemplifica os benefícios da aprendizagem baseada em projetos para capacitar os alunos a transferir seus conhecimentos e habilidades para problemas do mundo real, além de motivarem-se para aprender e melhorar seu desempenho em Matemática e Ciências. Suas pesquisas mostram que a aprendizagem baseada em projetos atende a essa necessidade e fornece o roteiro para que professores adotem uma abordagem bem sucedida.

É nessas perspectivas que a presente pesquisa se ancora ao desenvolver projetos de aprendizagem com o tema da robótica educacional. Perspectivas que associam os processos educacionais na busca por avanços e por soluções de problemas ligados ao desenvolvimento tecnológico.

A robótica educativa propõe uma associação do formalismo teórico escolar com projetos práticos, essencialmente interdisciplinares, envolvendo Matemática, Física, Lógica, dentre outras (SCHONS et al., 2010). A robótica possibilita que o estudante/pesquisador reflita sobre a essência dos problemas, promovendo o estudo de conceitos multidisciplinares, estimulando a busca por soluções estratégicas. Segundo os autores, essas características tornam a robótica uma ferramenta educacional de alto potencial de aprendizagem e envolvimento do aluno.

Nessa perspectiva, a pesquisa com objetivos de planejar e testar trajetórias de deslocamento suave para Micro Servo Motores acionados e controlados via plataforma Arduino, baseia-se em alguns pressupostos teóricos de mecânica e controle de robôs modernos. A obtenção e a implementação de trajetórias deve levar em consideração a minimização de erros com base na discussão das condições iniciais das variáveis de estado de sistemas dinâmicos. Essa trajetória, além de ser factível, deve ser computacionalmente tratável. A trajetória deve ser uma função suficientemente suave do tempo e deve respeitar qualquer limite dado nas velocidades, acelerações ou torques das juntas. (LYNCH; PARK, 2017, p. 325).

Trajetórias em salto, tipo degrau de posição, simplificam o problema de rastreamento da trajetória, mas mostram-se desaconselháveis devido à descontinuidade da velocidade e aceleração, sofrendo um maior estresse e produzindo perturbações. Trajetórias trapezoidais são obtidas a partir de trechos de aceleração máxima no início e no final dos trechos de deslocamento. São de difícil implementação pois necessitam de algum tipo de interpolação nos pontos de descontinuidade da 
aceleração, o que complica a implementação e reduz o desempenho deste tipo de trajetória (ANDERSSON, 1989). Trajetórias de tempo mínimo impõem velocidades mais altas possíveis a cada instante e ainda devem satisfazer as restrições de aceleração e de limite final do atuador. Essas trajetórias são bastante descontínuas em aceleração e necessitam a implementação de algoritmos de otimização numérica, sendo portanto computacionalmente dispendiosas (SHILLER; DUBOWSKY, 1991).

Trajetórias Polinomiais são percursos definidos por funções polinomiais entre dois pontos de deslocamento. São escolhas convenientes para representação de trajetórias porque suas propriedades são bem conhecidas e suas derivadas podem ser calculadas facilmente e são também polinômios. Trajetórias lineares são problemáticas porque implicam velocidade constante e consequentemente acelerações descontínuas nos instantes iniciais e finais.

A suavização de trajetórias que resulta em uma maior eficácia é a definição de trajetórias polinomiais de terceiro e quinto graus em relação ao tempo, pois permitem que restrições de velocidade e aceleração sejam satisfeitas (LYNCH; PARK, 2017, p. 328). A trajetória polinomial de terceiro grau permite satisfazer as restrições de posição e velocidade, e a de quinto grau, permite também satisfazer as condições de aceleração necessárias para uma suavização ótima.

Adicionalmente, é conveniente que a trajetória também seja contínua em aceleração, pois trajetórias que apresentam jerk (derivada da aceleração) elevado tendem a excitar ressonâncias mecânicas no manipulador, causando vibrações (CRAIG, 1989). Splines cúbicos também são possíveis, no entanto, esse tipo de trajetória utiliza vários segmentos, enquanto um único polinômio de quinto grau pode representar a trajetória inteira (SHILLER; DUBOWSKY, 1991).

\section{Metodologia}

O conhecimento científico passou a ser mais formalmente organizado a partir do século XVI, com Galileu Galilei por meio da discussão racional de um fenômeno ou de um objeto de investigação e da definição de um método para a realização dessa investigação. Decorre dessa organização uma série de metodologias com diferentes caracterizações. No caso deste trabalho, a escrita deste texto pode ser definida como pesquisa aplicada, uma vez que seus objetivos estão voltados para a resolução de problemas, visando alcançar contribuições para fins práticos.

A primeira etapa do processo consistiu na investigação para a obtenção de trajetórias polinomiais de deslocamento entre dois pontos de mecanismos móveis acionados e controlados por meio da plataforma Arduino. Nesse processo, foi utilizado o software de processamento numérico Scilab 5.5.2 para simulação das trajetórias, considerando-se, principalmente, as condições iniciais e finais de posição, velocidade e aceleração em cada trecho. O Scilab é um software de licença aberta, que permite a construção de rotinas para computação numérica com uma linguagem de programação de alto nível, orientada à análise numérica. 
A etapa seguinte consistiu na construção de uma pequena bancada de testes, incluindo uma placa de Arduino Uno, um Micro Servo Motor 9g Tower Pro SG90. Arduino é uma plataforma de prototipagem eletrônica de hardware livre, com linguagem de programação é a $\mathrm{C} / \mathrm{C}++$ e foi criada na Itália com o intuito de levar a programação para as pessoas de forma simples e com pouco custo, e sua. Servo Motores são máquinas, mecânicas ou eletromecânicas, que apresentam movimento proporcional a um comando, em vez de girarem ou se moverem livremente sem um controle mais efetivo de posição como a maioria dos motores. São dispositivos de malha fechada, ou seja, recebem um sinal de controle, verificam a posição atual, atuam no sistema indo para a posição desejada, movendo-se em graus, com amplitude nominal de giro de 0 a 180. A Figura 1, a seguir, mostra a placa de Arduino Uno e o Micro Servo Motor $9 \mathrm{~g}$ utilizados para os testes.

Figura 1 - Placa de Arduino Uno e Micro Servo Motor 9g.

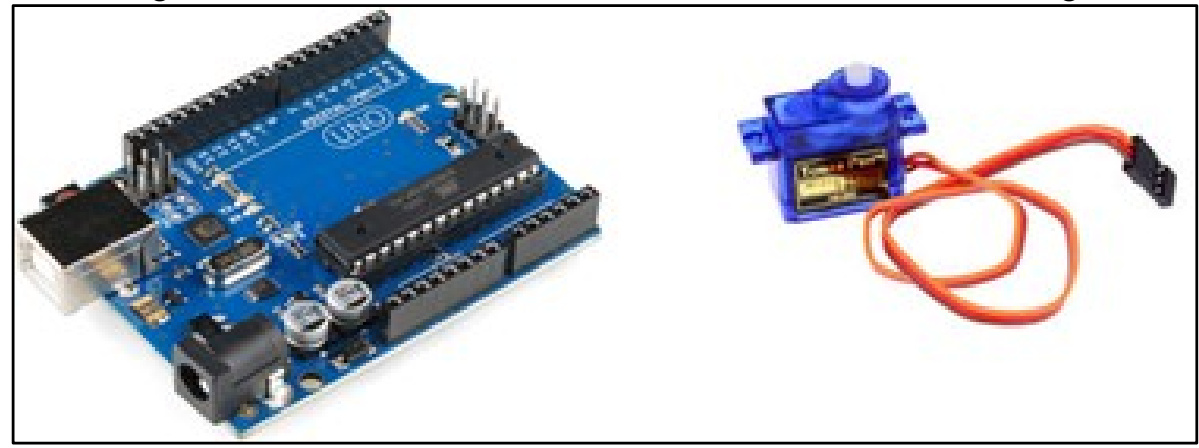

Fonte: www.filipeflop.com.br. Acesso em: 17 mar. 2018.

Para a fixação do Micro Servo Motor à bancada, foi confeccionada uma cápsula projetada para tal função por meio de uma impressora 3D. Por fim, também com uso de uma impressora 3D, foi confeccionada uma haste medindo $8 \mathrm{~cm}$ de comprimento que foi fixada ao eixo de giro do motor para acompanhar seu deslocamento semicircular.

Os testes experimentais tiveram como objetivo principal confrontar o desempenho do motor quanto ao seguimento de diferentes trajetórias predefinidas e, também, com relação ao posicionamento preciso em pontos predeterminadas. Para o acompanhamento do movimento executado pelo Micro Servo Motor, foi utilizada a metodologia de filmagem e posterior captura de dados relativos à posição em cada instante de tempo, possibilitando, com isso, comparação com a simulação numérica das trajetórias.

Uma limitação que precisou ser superada foi a de identificar o momento exato de início e de fim dos comandos para o deslocamento do Micro Servo Motor. Para isso, a estratégia utilizada foi a instalação de um ponto de led na extremidade da haste. O led pode ser acionado no instante de início do deslocamento da haste e desligado no instante final do comando, permitindo a identificação precisa do movimento.

Para o processo de filmagem, foi utilizada uma Câmera Canon Eos Rebel T6 com Lente 18$55 \mathrm{~mm}$, a qual permite filmagem em alta definição com até 60 frames por segundo, diferentemente 
da maioria das câmeras caseiras, ou de celulares que alcançam, no máximo 24 frames por segundo. A Figura 2 mostra, no primeiro quadro, a captura de vídeo em testes experimentais e, no segundo quadro, a bancada de testes utilizada com detalhamento dos componentes.

Figura 2 - Bancada de testes utilizada.

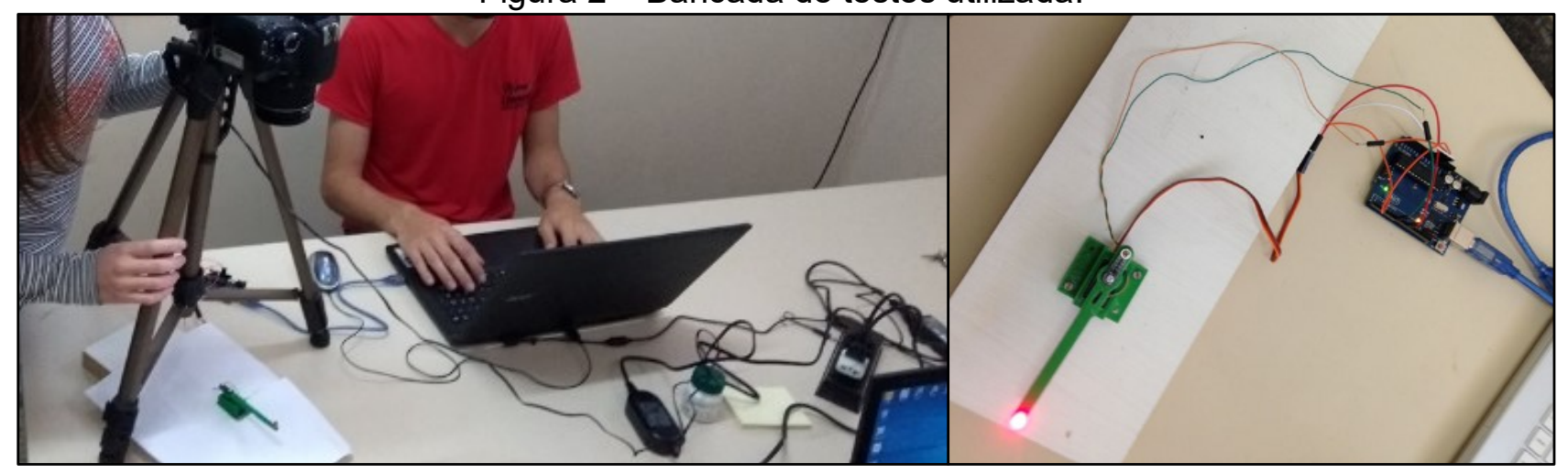

Fonte: Arquivo dos autores.

Para a captura dos dados da relação tempo/posição do giro do motor, foi utilizado o software Tracker. O Tracker é uma aplicação gráfica em Java publicada mediante a licença GPL que permite analisar vídeos do ponto de vista físico. Com o uso desse software, é possível obter dados precisos com relação ao movimento de giro do motor e exportar tais dados para processamento e análise no Scilab. A figura 3, a seguir, mostra a captura de dados por meio do vídeo no software Tracker, tomando como referencial o ponto de led na extremidade da haste.

Figura 3 - Interface do software Tracker durante a captura de dados.

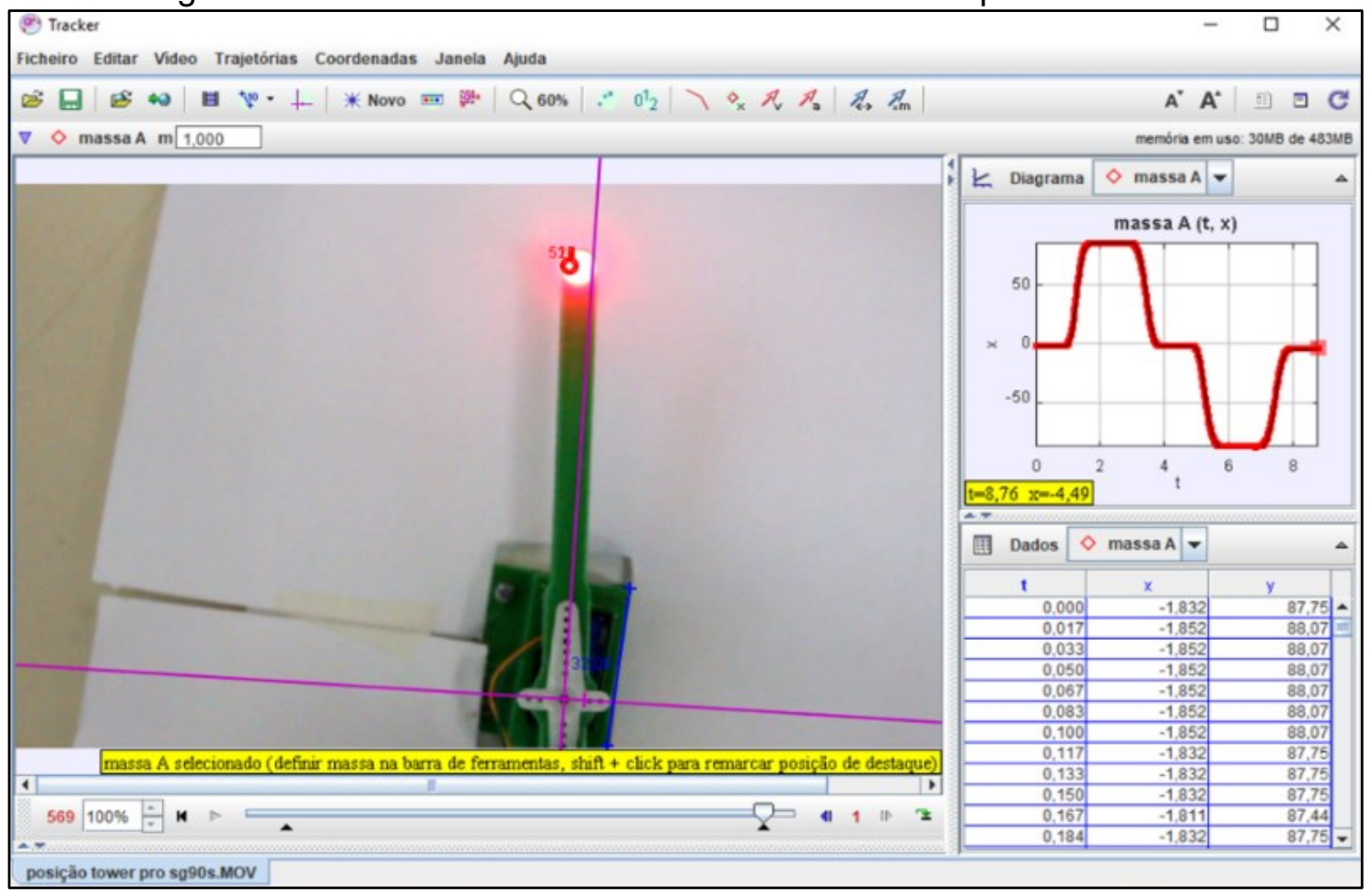

Fonte: Arquivo dos autores. 


\section{Resultados e Discussões}

O movimento realizado pelo Micro Servo Motor utilizado na pesquisa resulta do giro de seu eixo, dado em graus, com amplitude nominal de 0 a $180^{\circ}$. O planejamento de trajetórias para esse tipo de atuadores consiste no deslocamento de uma posição inicial $\theta_{0}$ até uma posição final $\theta_{f}$. $O$ comando mais comum para execução de movimento, utilizado em rotinas de controle de mecanismos autônomos com esse tipo de atuador, é o deslocamento em salto, ou seja, se o mecanismo está em uma determinada posição $\theta_{1}$, deve deslocar-se imediatamente para uma posição $\theta_{2}$, sem reserva de intervalo de tempo para esse movimento.

Com base nessa prática, a primeira trajetória planejada foi uma trajetória em salto com testes de parada no centro e próximo às duas extremidades. Considerando-se a amplitude nominal do Servo Motor e evitando os pontos extremos, a trajetória previa posicionamento inicial na posição central, ou seja, $\theta_{0}=90^{\circ}$, seguindo para a posição $\theta_{1}=15^{\circ}$, posteriormente voltando para $\theta_{2}=90^{\circ}$, movendo-se em seguida para $\theta_{3}=165^{\circ} \mathrm{e}$, finalmente, retornando para $\theta_{4}=90^{\circ}$, permanecendo parado, em todos os pontos, por 1000 milissegundos.

Desse modo, sendo $P(t)$ a posição em graus a ser ocupada pelo atuador em função do tempo, essa trajetória é dada por:

$$
P(t)=\left\{\begin{array}{l}
90, \text { se } \quad 0 \leq t<1000 \mathrm{~ms} \\
15, \text { se } 1000 \leq t<2000 \mathrm{~ms} \\
90 \text {, se } 2000 \leq t<3000 \mathrm{~ms} \\
165 \text {, se } 3000 \leq t<4000 \mathrm{~ms} \\
90 \text {, se } \quad 4000 \leq t \leq 5000 \mathrm{~ms}
\end{array}\right.
$$

Foram realizados testes para essa trajetória, cujo resultado é mostrado na Figura 4, a seguir. Observa-se um desempenho aceitável com relação ao posicionamento, com imprecisões mais significativas nas extremidades. No entanto, observa-se a necessidade de um tempo significativo para deslocamento de uma posição a outra, de tal modo que esse tipo de trajetória torna-se naturalmente inadequado para movimentos que exigem seguimento de trajetória em deslocamento contínuo. 


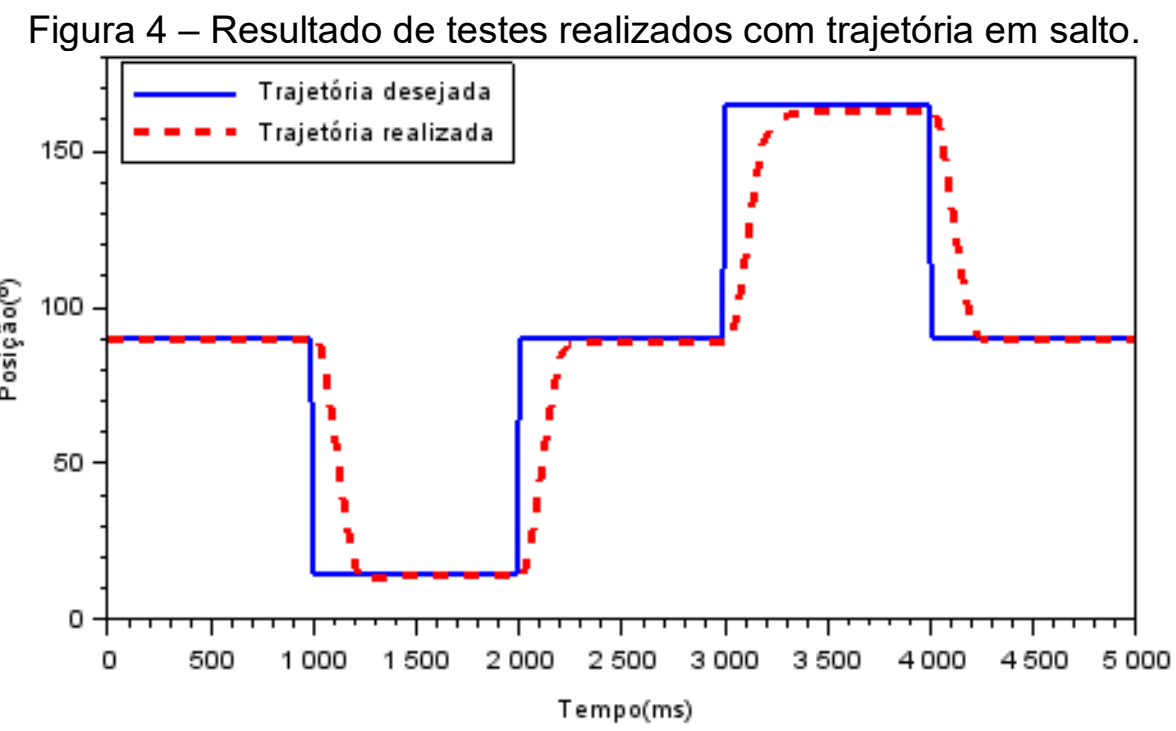

Fonte: Elaboração dos autores.

Com o intuito de permitir o seguimento de trajetória com deslocamento contínuo, foi definido um tempo de 1000 milissegundos para deslocamento de um ponto a outro, mediante uma trajetória linear dada por uma função de primeiro grau. A trajetória previa o deslocamento da posição $P(0)=$ $90^{\circ}$ até a posição $P(1000)=15^{\circ}$, desse modo, para uma função polinomial de primeiro grau do tipo $P(t)=a t+b$, obtém-se $a=-0,075$ e $b=90$, ou seja:

$$
P(t)=-0,075 t+90
$$

Por meio de translações verticais e horizontais, além da inversão vertical do percurso, é possível obter a trajetória completa de deslocamento com testes de parada e com trechos de seguimento contínuo por meio do polinômio de primeiro grau. Essa trajetória é dada por:

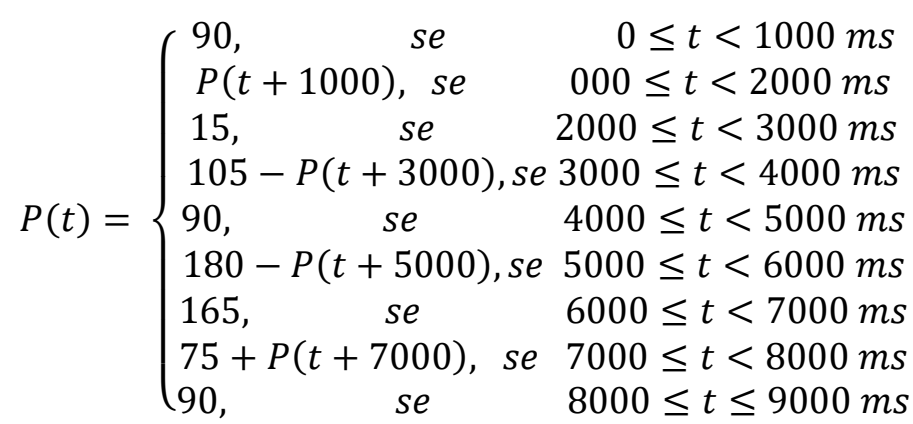

Os resultados dos testes experimentais para essa trajetória são mostrados na Figura 5, a seguir, incluindo, no primeiro quadro, a comparação entre trajetória desejada e trajetória realizada e, no segundo quadro, o erro resultante dessa comparação. Observa-se que, mesmo havendo atrasos no deslocamento, é possível a realização de trajetórias de deslocamento contínuo com seguimento de trajetória entre os pontos de parada. 
Figura 5 - Resultado de testes realizados com trajetória polinomial de primeiro grau.
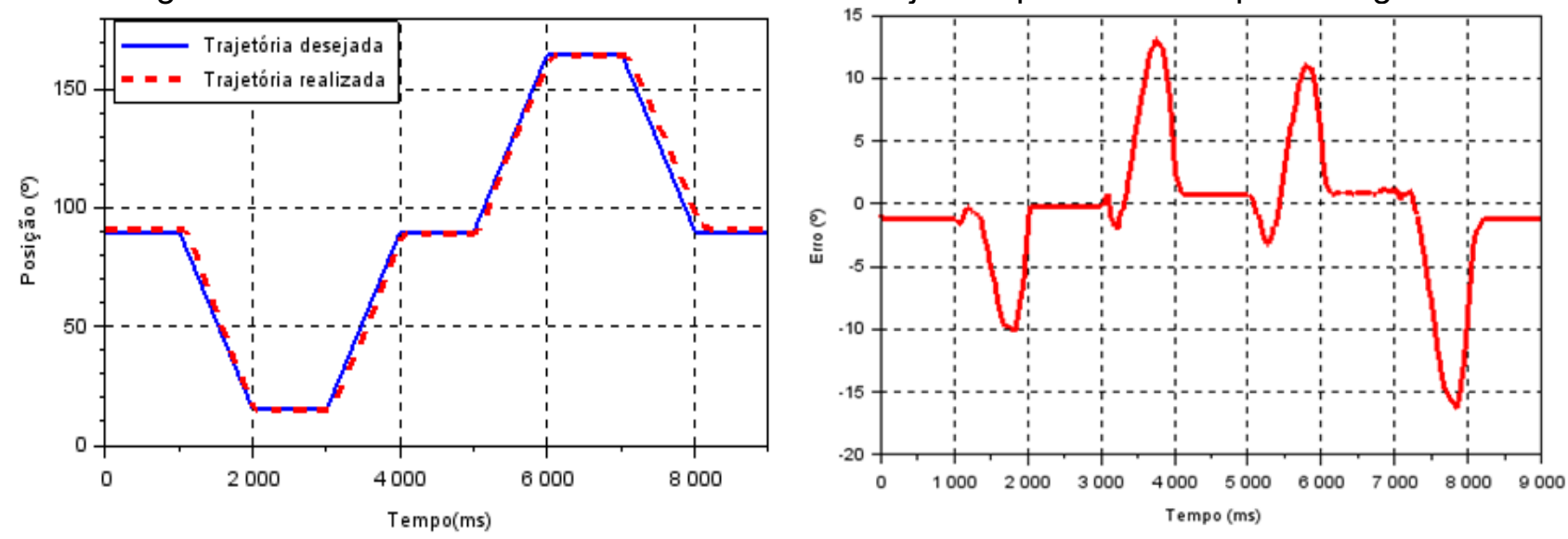

Fonte: Elaboração dos autores.

A trajetória polinomial de primeiro grau resulta num movimento brusco no início do trecho de deslocamento e parada brusca no final, fato que resulta em erros elevados. Isso ocorre porque o movimento já se inicia com velocidade diferente de zero. Com o intuito de melhorar o desempenho e procurando evitar esse início brusco do deslocamento, uma estratégia possível é a suavização da arrancada e da chegada do movimento por meio da definição da velocidade inicial nula nas extremidades. Como a velocidade é dada pela derivada da função deslocamento, é possível estabelecer quatro condições a serem satisfeitas por essa função deslocamento. Desse modo, obtém-se um sistema linear de quarta ordem dado por:

$$
\left\{\begin{array}{l}
P\left(t_{0}\right)=a t_{0}{ }^{3}+b t_{0}{ }^{2}+c t_{0}+d \\
P^{\prime}\left(t_{0}\right)=3 a t_{0}^{2}+2 b t_{0}+c \\
P\left(t_{f}\right)=a t_{f}{ }^{3}+b t_{f}^{2}+c t_{f}+d \\
P^{\prime}\left(t_{f}\right)=3 a t_{f}^{2}+2 b t_{f}+c
\end{array}\right.
$$

no qual $t_{0}$ é o instante inicial do deslocamento e $t_{f}$ o instante final.

A solução desse sistema linear permite a determinação dos coeficientes de um polinômio de terceira ordem para deslocamento entre os dois pontos e com velocidade nula nas extremidades, ou seja, para $P(t)=a t^{3}+b t^{2}+c t+d$, com $P(0)=90, P(1000)=15, P^{\prime}(0)=0$ e $P^{\prime}(1000)=0$ obtém-se $a=1,5 \times 10^{-7}, b=-2,25 \times 10^{-4}, c=0$ e $d=90$. Utilizando o mesmo esquema da Equação 3 para o polinômio de terceira ordem, o resultado dos testes experimentais realizados são mostrados na Figura 6, a seguir, incluindo, no primeiro quadro, a comparação entre trajetória desejada e trajetória realizada e, no segundo quadro, o erro resultante dessa comparação. 
Figura 6 - Resultado de testes realizados com trajetória polinomial de terceiro grau.
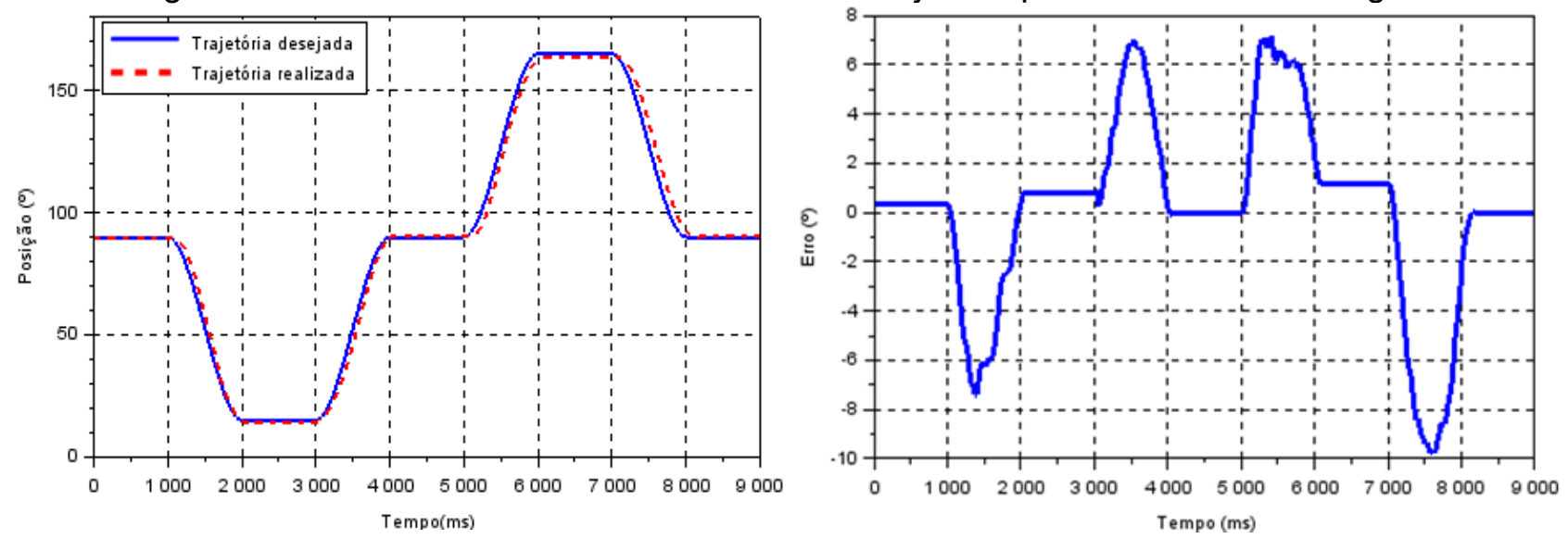

Fonte: Elaboração dos autores.

A trajetória de terceiro grau testada resultou em uma redução significativa do erro de seguimento de trajetória nos trechos de deslocamento entre dois pontos de parada, devido à suavização no início e no fim desses trechos. Com o intuito de minimizar ainda mais os erros seguimento de trajetória, foi adotada a estratégia de definir a aceleração também nula no ponto de partida e no ponto de chegada dos trechos. Com isso, além das condições iniciais da trajetória de terceiro grau, acima descrita, foi considerada a derivada segunda igual a zero nas duas extremidades, ou seja, $P^{\prime \prime}(0)=0$ e $P^{\prime \prime}(1000)=0$. Desse modo, é possível definir um sistema linear de sexta ordem e determinar os coeficientes de um polinômio de quinto grau do tipo $P(t)=a t^{5}+$ $b t^{4}+c t^{3}+d t^{2}+e t+f$ com velocidade e aceleração nula nas extremidades. A solução desse sistema define $a=-4,5 \times 10^{-13}, b=1,125 \times 10^{-9}, c=-7,5 \times 10^{-7}, d=0, e=0$ e $f=90$.

Utilizando-se o mesmo esquema da Equação 3 para o polinômio de quinto grau, o resultado dos testes experimentais realizados é mostrado na Figura 7, incluindo, no primeiro quadro, a comparação entre trajetória desejada e trajetória realizada e, no segundo quadro, o erro resultante dessa comparação.

Figura 7 - Resultado de testes realizados com trajetória polinomial de quinto grau.
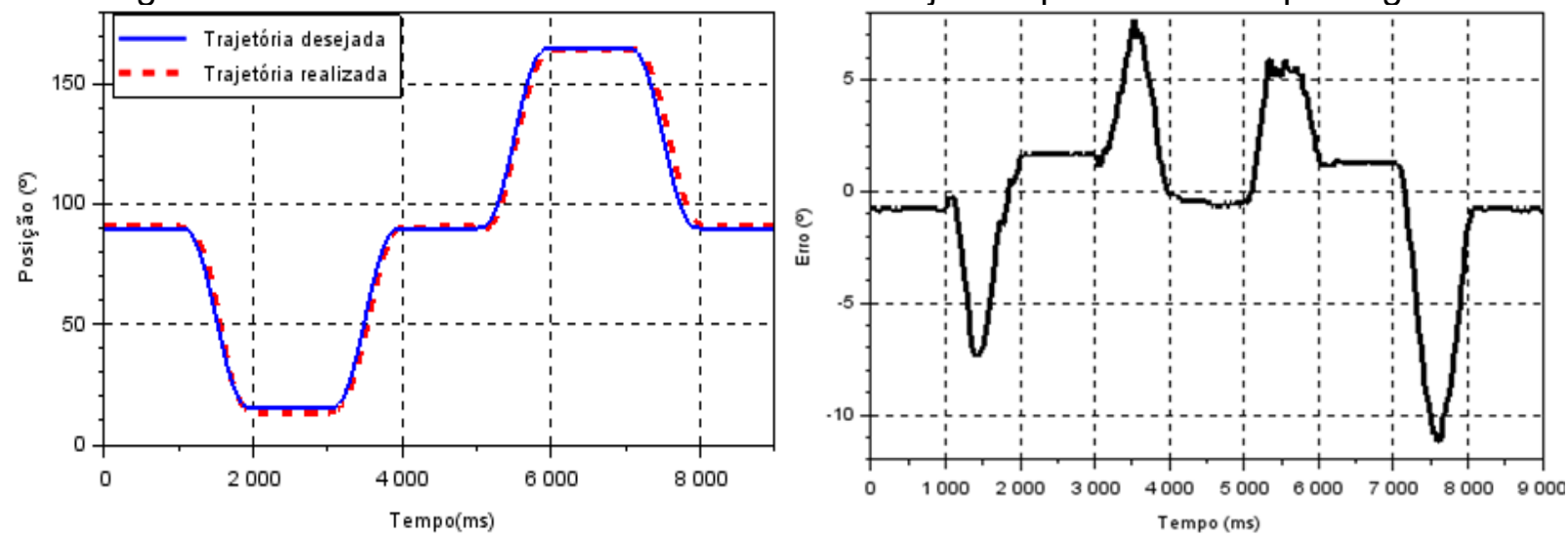

Fonte: Elaboração dos autores. 
A análise dos gráficos de erros das trajetórias de terceiro e quinto grau mostra um desempenho semelhante, sem redução significativa de erros. A Figura 8, a seguir, mostra um comparativo entre os erros resultantes da implementação das três trajetórias polinomiais de deslocamento suave.

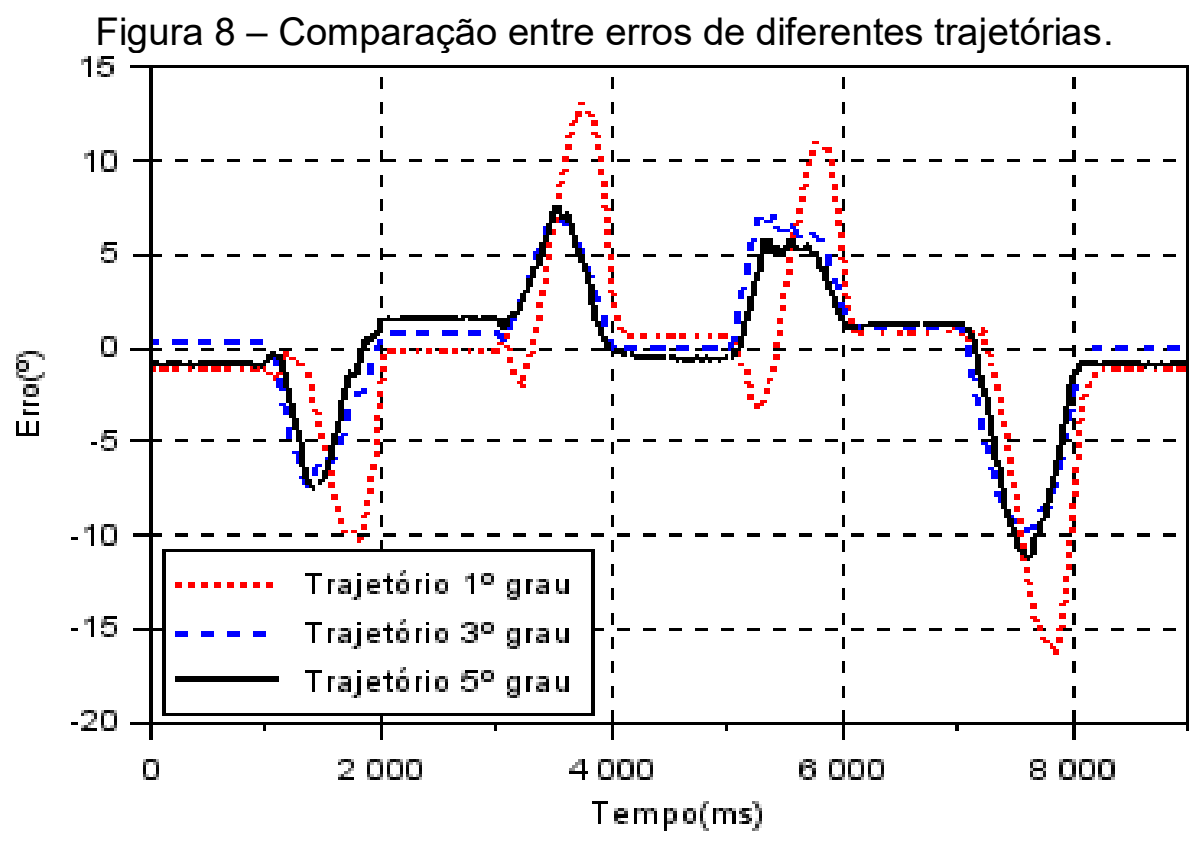

Fonte: Elaboração dos autores.

Com base na análise do comparativo de erros da Figura 8, observam-se desempenhos muito semelhantes entre as trajetórias de terceiro e quinto grau. Por outro lado, a capacidade de processamento exigida para a implementação da trajetória de quinto grau é significativamente alta, considerando-se a capacidade limitada para a plataforma Arduino que opera o sistema. Desse modo, conclui-se que a trajetória de terceiro grau é a mais adequada para esse tipo de aplicação.

\section{Considerações Finais}

A pesquisa sobre planejamento de trajetórias polinomiais para robótica com Arduino que culminou com a escrita deste trabalho apresenta estratégias que permitem melhorar o desempenho de Micro Servo Motores em aplicações à robótica. A definição de trajetórias polinomiais que suavizam o deslocamento reduz significativamente erros de seguimento de trajetória para esse tipo de atuador. A metodologia utilizada, principalmente quanto à montagem de uma bancada de testes com a instalação de um ponto de led na extremidade da haste, permitiu identificar com precisão o momento exato de início e fim dos comandos para o deslocamento do Micro Servo Motor. Com essa estratégia e com uso o de uma filmadora que captura 60 frames por segundo, foi possível acompanhar precisamente o movimento gerado e, assim, avaliar o desempenho do Servo Motor em cada situação. 
Testadas as trajetórias polinomiais de primeiro, terceiro e quinto grau, a trajetória de terceiro grau resultou numa significativa redução de erros em relação à de primeiro grau, sobretudo pela utilização da estratégia de determinação da velocidade nula nos extremos do deslocamento. Por outro lado, a trajetória de quinto grau, que considera também a aceleração nula nas extremidades, apresentou resultados semelhantes à de terceiro grau.

O desenvolvimento de trajetórias de suavização de movimento para robótica com Arduino não está presente como tema de pesquisas recentes, ressaltando a originalidade do presente trabalho. Além disso, essas estratégias podem ser aplicadas em diferentes projetos de robótica, até mesmo na indústria, melhorando o desempenho de atuadores e aprimorando a execução de tarefas autônomas.

O desenvolvimento da pesquisa com base nos pressupostos teórico-metodológicos de STEM e de Aprendizagem Baseada em Projetos possibilitou o envolvimento de estudantes na busca por soluções de problemas reais. Nesse processo, os estudantes desenvolvem seus estudos e pesquisas criando, planejando, desenvolvendo e implementando projetos interdisciplinares que possuam fins essenciais para a vida em sociedade e para seu futuro.

\section{Referências}

ANDERSSON, R. L. Aggressive trajectory generator for a robot ping-pong player. IEEE Control Systems Magazine, v. 9, n. 1, p. 15-21, fev. 1989.

ARDUINO. Home Page. Disponível em: <https://www.arduino.cc/>. Acesso em: 15 mar. 2018.

BENDER, W. N. Aprendizagem Baseada em Projetos: Educação Diferenciada para o Século XXI. Porto Alegre: Penso Editora, 2014.

BYBEE, R. W. The Case for STEM Education: Challenges and Opportunities. Arlington: NSTA Press, 2013.

COBB, P.; CONFREY, J.; DISESSA, A.; LEHRER, R.; SCHAUBLE, L. Design Experiments in Educational Research. Educational Researcher, v. 32, n. 1, p. 9-13, jan./fev. 2003.

CRAIG, J. J. Introduction to Robotics Mechanics and Control. 2. ed. Addison-Wesley, 1989.

DOERR, H. M.; WOOD, T. Pesquisa-Projeto (design research): aprendendo a ensinar Matemática. In: BORBA, M. C. (Org.). Tendências internacionais em formação de professores de matemática. Belo Horizonte: Autêntica, 2006. p. 113-128.

ENGLISH, L. D. STEM education K-12: perspectives on integration. International Journal of STEM Education, p. 1-8, 2016.

LABOY-RUSH, D. Integrated STEM Education through Project-Based Learning. In Learning.com. 2013. Disponível em:

<https://www.rondout.k12.ny.us/common/pages/DisplayFile.aspx?itemld=16466975>. Acesso em: 15 mar. 2018.

LYNCH, M. K.; PARK, F. C. Modern Robotics Mechanics, Planning, and Control. Cambridge University Press, 2017. 
POPOV, E. P.; YUREVICH, E. I. Robotics. Moscow: Mir Publishers, 1987.

SANCHEZ, W. A quarta revolução industrial e seus impactos na educação. Disponível em: <https://blog.abmes.org.br/?p=11915>. Acesso em: 11 mar. 2018.

SCHONS, C.; PRIMAZ, E.; WIRTH, G. Introdução a Robótica Educativa na Instituição

Escolar. 2010. Disponível em: <https://www.yumpu.com/pt/document/view/3530407/introducao-arobotica-educativa-nainstituicao-escolar-para>. Acesso em: 19 mar. 2018.

SCHWAB, K. A Quarta Revolução Industrial. 1. ed. Editora EDIPRO, 2016.

SHILLER, Z.; DUBOWSKY, S. On computing the global time-optimal motion of robotic manipulators in the presence of obstacles. IEEE Transactions on Robotics and Automation, $v$. 7, n. 6, p. 785-797, dez. 1991.

SILVA, S. R. X. Protótipo De um Robô Móvel Interdisciplinar de Baixo Custo para uso educacional em Cursos Superiores de Engenharia e Computação. 2011. 220 f. Dissertação (Mestrado em Mecatrônica) - Universidade Federal da Bahia, Salvador, 2011.

VAZ, R. L. O uso das isometrias do Software Cabri-Gèométre como recurso no processo de prova e demonstração. 2004. 216 f. Dissertação (Mestrado em Educação) - Pontifícia Universidade Católica de São Paulo, São Paulo, 2004.

VENTURELLI, M. A tecnologia ignora a crise: A Automação Industrial provocando a $4^{\text {a }}$ Revolução Industrial. Automação Industrial, ago. 2017. Disponível em:

$<$ https://www.automacaoindustrial.info/tecnologia-ignora-crise-automacao-industrial-provocando4a-revolucao-industrial/>. Acesso em: 22 mar. 2018. 